\title{
A MODULAR TOOL FOR THE EVOLUTIONARY SYNTHESIS OF STELLAR POPULATIONS
}

\author{
CLAUDIA MARASTON \\ Dipartimento di Astronomia, Universitdi Bologna \\ Via Zamboni 33, 40126 Bologna, Italy
}

An innovative tool for the construction of Evolutionary Synthesis models of Stellar Populations is presented. It is based on three independent matrices giving respectively 1 ) the fuel consumption during each evolutionary phase as a function of stellar mass, 2) the typical temperatures and gravities during such phases, and 3) colors and bolometric corrections as a function of gravity and temperature. The first matrix allows to calculate the relative contribution of each phase to the bolometric light of the population, thanks to the so-called Fuel Consumption Theorem (Renzini \& Buzzoni 1986, in Spectral Evolution of Galaxies, ed. C. Chiosi and A. Renzini (Dordrecht:Reidel),p.195)). The other two matrices allow to distribute such contributions in the various passbands, and thus to compute the synthetic colors of the population as a function of age. The modular structure of the code allows to easily assess the impact on the synthetic spectral energy distribution of various model ingredients (e.g. uncertainties in the stellar evolutionary models, mixing length, temperature distribution of the horizontal branch (HB) stars, AGB mass loss, color-temperature transformations, etc.) Models have been computed with $\mathrm{Y}=0.27, \mathrm{Z}=0.02$ and for three choices of a double-slope IMF ( $s=1+x=2.35,3.5,1.5$ for $M \geq 0.6 M_{\odot}$ and $s^{\prime}=s-2.5$ for $\left.\mathrm{M}<0.6 M_{\odot}\right)$. Among the main results, it is found that: i) the appearence of an extended AGB has a strong impact on the integrated $(V-K)_{0}$ color, which increases by $\simeq 1.5 \mathrm{mag}$ in $\Delta t \simeq$ $400 \mathrm{Myr}$; ii) the inclusion of a blue-HB developing at ages around $10 \mathrm{Gyr}$ produces a hook-shape in the $(U-B)_{0}$ vs $(B-V)_{0}$. locus described by aging stellar populations, in agreement with what observed for Magellanic Clouds Clusters.

A detailed description of the variuos results will be given in a forthcoming paper. 